\title{
Surgeon's core values: legitimizing the team concept in surgery
}

\author{
Jason Park and Peter T Scardino
}

Ethical models of professional responsibility in surgery have traditionally been highly individualistic. Patient ownership is accepted as part of the surgical culture, with an expectation that surgeons individually care for, and attend to, their own patients. A recent qualitative study of surgeons and surgical residents in academic settings (Park et al. [2007] Surgery 142: 111-118), however, found professional responsibility to be a much more complex construct, with an evolving perception of patient care as a collective responsibility. The responsibility for patient care was largely enacted through teamwork, with surgeons and residents referencing the overarching concept of teamwork and specific factors that were considered an integral part of the teamwork process, including adherence to on-call schedules, safe and efficient mechanisms to transfer patient care responsibilities, effective communication between team members, and the development of a trusting relationship with colleagues.

The teamwork concept in surgery is more than just a theory; there is a growing body of literature suggesting that poor teamwork and communication are strongly associated with adverse events in the clinical setting. Gawande et al. (Surgery [2003] 133: 614-621) analyzed errors reported by surgeons, and found that communication failure was a factor in $43 \%$ of errors made in surgery. Furthermore, Lingard et al. (Qual Saf Health Care [2004] 13: 330-334) found that communication failures occurred in approximately $30 \%$ of team exchanges in the operating room, which had negative effects on systems processes, such as procedural errors, and led to inefficiency and delays, wasted resources, and tension within the team. The findings from these studies have important implications for patient care, as these errors lead not only to inconvenience or delays in care, but also have the potential to cause serious adverse clinical events or lead to 'near misses'. ....there is a

growing body

of literature

suggesting

that poor

teamwork and

communication

are strongly

associated

with adverse

events in the

clinical setting.

J Park is a Fellow in

Surgical Oncology,

Memorial Sloan-

Kettering Cancer

Center, New York,

NY, and PT Scardino

is Editor-in-Chief,

Nature Clinical

Practice Urology.

Competing interests

The authors declared no

competing interests.

www.nature.com/clinicalpractice doi:10.1038/ncpuro0973
Despite its critical relationship to safety in healthcare, the team concept in day-to-day surgical practice has not reached the formal and standardized level that it has in other professions or industries, most notably the aviation industry. Teamwork and key aspects of the teamwork process, such as communication, are developed in more informal and tacit manners in medical teams, and often are practiced unsystematically. Surgical teams are expected to perform at a high standard, but formal programs to teach team proficiency or monitor team function have not been established at most institutions.

Evolving healthcare practices suggest that there is a need to reframe professional ethics in surgery from individualism to responsibility in a collective sense. Teamwork should be considered a core trait of surgical professionalism and a fundamental part of organizational practice. First, the collective responsibility to patient care should be made an explicit and formal part of the professional code in surgery. Second, there is a legitimate need to develop and implement educational interventions that define teamwork models and promote collaborative behavior in surgery. Current areas of interest include programs such as Crew Management Resource Training, which teaches participants to function as parts of larger, service-oriented structures. Third, systems development is necessary to facilitate teamwork processes, ranging from practice changes that formalize sign-out procedures to environmental changes that ensure ready access to medical records. Finally, we need to develop and implement measures that evaluate educational interventions and monitor progress, both in terms of team function and overall clinical outcomes. The process of legitimizing the surgical team and improving team function presents definite challenges. Rather than allowing this process to unfold on an ad hoc basis, we should embrace the opportunity to make positive changes and optimize the care of the modern surgical patient. 